\title{
Determining the mechanical constitutive properties of metals as a function of strain rate and temperature: A combined experimental and modeling approach
}

Development and validation of constitutive models for polycrystalline materials subjected to high strain rate loading over a range of temperatures are needed to predict the response of engineering materials to in-service type conditions (foreign object damage, high-strain rate forging, high-speed sheet forming, deformation behavior during forming, response to extreme conditions, etc.). To account accurately for the complex effects that can occur during extreme and variable loading conditions, requires significant and detailed computational and modeling efforts. These efforts must be closely coupled with precise and targeted experimental measurements that not only verify the predictions of the models, but also provide input about the fundamental processes responsible for the macroscopic response. Achieving this coupling between modeling and experimentation is the guiding principle of this program. Specifically, this program seeks to bridge the length scale between discrete dislocation interactions with grain boundaries and continuum models for polycrystalline plasticity. Achieving this goal requires incorporating these complex dislocation-interface interactions into the well-defined behavior of single crystals. Despite the widespread study of metal plasticity, this aspect is not well understood for simple loading conditions, let alone extreme ones.

Our experimental approach includes determining the high-strain rate response as a function of strain and temperature with post-mortem characterization of the microstructure, quasi-static testing of pre-deformed material, and direct observation of the dislocation behavior during reloading by using the in situ transmission electron microscope deformation technique. These experiments will provide the basis for development and validation of physically-based constitutive models, which will include dislocation-grain boundary interactions for polycrystalline systems. One aspect of the program will involve the direct observation of specific mechanisms of micro-plasticity, as these will indicate the boundary value problem that should be addressed. This focus on the pre-yield region in the quasi-static effort (the elasto-plastic transition) is also a tractable one from an experimental and modeling viewpoint. In addition, our approach will minimize the need to fit model parameters to experimental data to obtain convergence. These are critical steps to reach the primary objective of simulating and modeling material performance under extreme loading conditions.

To achieve these goals required assembling a multidisciplinary team, see Table 1 , with key collaborators at the National Laboratories. One of the major issues for the team members was to learn about the expertise available and how to communicate across disciplines. The communication issue is a challenging one and is being addressed in part with weekly meetings in which the graduate students present lectures on the fundamentals of their respective areas to the entire group. Breakthroughs in science are presented but these, by necessity, assume a tutorial nature. For example, interpreting electron micrographs and understanding what can be achieved by using electron microscopy is challenging for the modeling expert as is comprehending the input and limitations of crystal plasticity codes for an electron microscopist. Significant progress has been made at dissolving these barriers and the students are able to work across the disciplines. 
Table 1. Project participants, Affiliations and Percentage of time supported by this grant.

\begin{tabular}{|c|c|c|}
\hline Principal Investigators & Department Affiliation & $\begin{array}{l}\text { Fraction of time } \\
\text { supported by this } \\
\text { grant }\end{array}$ \\
\hline Professor I. Robertson, & Materials Science and Engineering (MatSE) & 0.5 month \\
\hline Professor A. Beaudoin & Mechanical and Industrial Engineering (MIE) & 1 month \\
\hline Professor John Lambros & $\begin{array}{l}\text { Aeronautical and Astronautical Engineering } \\
\text { (AAE) }\end{array}$ & 1 month \\
\hline \multicolumn{3}{|c|}{ Graduate Students } \\
\hline Cindy Smith & MSE & $100 \%$ \\
\hline Bryan Miller & MSE & $100 \%$ \\
\hline Jamie Kimberley & $\mathrm{AE}$ & \\
\hline Henry Padilla & MIE & $100 \%$ \\
\hline Satya Varadhan & MIE $^{*}$ & $100 \%$ \\
\hline Khaled Al-Fadhalah & $\mathrm{MIE}^{\S}$ & $20 \%$ (travel support) \\
\hline \multicolumn{3}{|c|}{$\begin{array}{l}\text { * Supported through a matching contribution from the ASCI Level } 1 \text { Center at UIUC. } \\
\S \text { Supported through the government of Kuwait. }\end{array}$} \\
\hline \multicolumn{3}{|c|}{ Collaborators at National Laboratories } \\
\hline David Lassila & \multicolumn{2}{|l|}{ Lawrence Livermore National Laboratory } \\
\hline Vassily Bulatov & \multicolumn{2}{|l|}{ Lawrence Livermore National Laboratory } \\
\hline Geoff Campbell & \multicolumn{2}{|l|}{ Lawrence Livermore National Laboratory } \\
\hline Carlos Tomé & \multicolumn{2}{|l|}{ Los Alamos National Laboratory } \\
\hline Amit Misra & \multicolumn{2}{|l|}{ Los Alamos National Laboratory } \\
\hline George Kaschner & \multicolumn{2}{|l|}{ Los Alamos National Laboratory } \\
\hline Dave Korzekwa & \multicolumn{2}{|l|}{ Los Alamos National Laboratory } \\
\hline
\end{tabular}

\section{Key achievements for this fiscal period.}

\section{Development of a crystal plasticity model that includes the effect of boundaries as a source, a barrier and a sink for dislocations.}

One of the challenges for plasticity codes, especially as progress is made toward a multiscale approach, is the transition from single to polycrystalline materials especially in the elasto-plastic transition region. Early work by Robertson ${ }^{1-4}$ and coworkers established experimentally the conditions for slip transfer across high-angle and random grain boundaries during the early stages of quasi-static loading. Specifically, it was determined that the slip transfer process was governed by competition between the slip system experiencing the maximum local resolved shear stress (applied stress + dislocation pile-up contribution) and the change in energy of the grain boundary as a consequence of the dislocation absorption and emission process. More recently these conditions have been shown applicable to interfaces between different materials or phases ${ }^{5,6}$. Hoagland et al. have demonstrated that the stress transmission effected by a semi-coherent (or nearly incoherent) bi-material boundary is affected by the core structure of misfit dislocations in the boundary. Spreading of the core can lead to a boundary that has increased mobility of misfit dislocations and concomitant weakening of the boundary in shear ${ }^{7}$. Hence, one must consider boundary structure on slip transfer. This is a complex problem as the boundary structure is not necessarily constant along the length of the grain boundary, creating weak and strong regions within the grain boundary. 
The challenge then is to formulate these slip transmission rules, at least in a phenomenological sense, into models that can be included in polycrystalline plasticity codes. One of the major achievements this fiscal period has been the development of appropriate models to account for slip transfer across interfaces. Basically, this involves accounting for the grain boundary by considering a bicrystal inclusion as the representative volume element of the aggregate. Each component of the bicrystal is subjected to a viscoplastic constitutive law and the response of the inclusion is determined based on the volume fraction of the components. Compatibility and equilibrium requirements are imposed across the interface. Interaction between the inclusions and the aggregate is accounted for through a visco-plastic self-consistent scheme. The grain boundary misorientation is accounted for by the bicrystal and the local stress distribution through the viscoplastic constitutive law. To account for the change in the grain boundary energy, we propose:

- development of a "latent hardening" applied to slip systems which intersect the boundary;

- using an effective "back stress" created as a result of the residual dislocation produced as the difference between the absorbed and emitted dislocations.

The first choice applies to boundaries with characteristics of high mobility of misfit dislocations such as at a bi-material interface, and the latter to slip transmission across grain boundaries, including twin boundaries. The predictions of the model are being tested against respective material systems and for different loading conditions. In the following, we outline development of the model, show the introduction of the latent hardening approach to $\mathrm{Cu} / \mathrm{Nb}$ nanolayers, and comment on our on-going experimental study of the effect of twin boundaries on deformation.

\section{A. Composite Grain Model}

The model is built upon the Viscoplastic Self-Consistent Code (VPSC) developed by Carlos Tomé at LANL. In general, each composite grain can consist of two crystals with different volume fraction. [For congruence with the subsequent example, the two phases are identified as $\mathrm{Cu}$ and $\mathrm{Nb}$.] The misorientation between the two crystals can be specific, i.e., Kurdjumov-Sachs orientation relationship, or random. The interface between the two crystals is assumed to be planar having its normal along the 3-direction (ND) as shown in Figure 1. The deformation response of both crystals is uniform, but not necessarily equal. The composite grain deformation follows the requirements of compatibility and equilibrium at the interface. In addition, one can determine the response of a composite grain by averaging the response of both crystals with respect to their volume fractions $\left(f^{C u} \& f^{N b}\right)$ such that the lattice deformation and rotation rate of the composite grain, $D^{c g}$ and $W^{c g}$, respectively, are expressed by:

$$
\begin{aligned}
& D^{c g}=f^{C u} D^{C u}+f^{N b} D^{N b} \\
& W^{c g}=f^{C u} W^{C u}+f^{N b} W^{N b} .
\end{aligned}
$$

Similarly, the deviatoric stress $\sigma^{\prime c g}$ is written:

$$
\sigma^{c g}=f^{C u} \sigma^{\prime c u}+f^{N b} \sigma^{N b} .
$$

The compatibility constraint requires continuous velocity across the interface. Therefore the following components of deformation rate are required to be equal at the interface: 


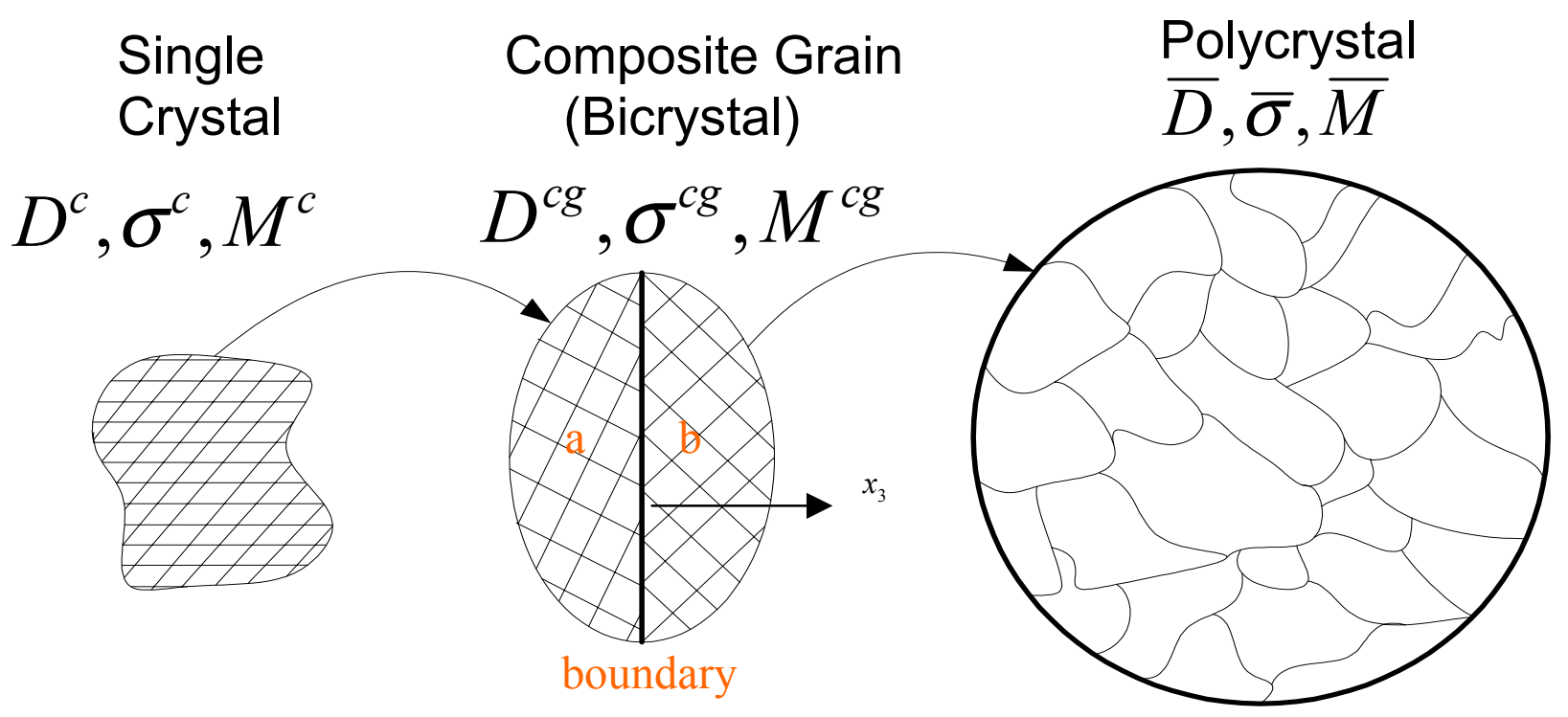

Figure 1 - Schematic of the composite grain model.

$$
D_{\alpha \beta}^{c g}=D_{\alpha \beta}^{C u}=D_{\alpha \beta}^{N b} \quad \alpha, \beta=1,2
$$

The other components are expressed by the averaging scheme:

$$
D_{3 \alpha}^{c g}=\left(f^{C u} D_{3 \alpha}^{C u}+f^{N b} D_{3 \alpha}^{N b}\right) \quad \alpha=1,2
$$

The requirement on the continuity of traction across the interface implies that only the following shear stress components are continuous:

$$
\sigma_{3 \alpha}^{\prime c g}=\sigma_{3 \alpha}^{\prime C u}=\sigma_{3 \alpha}^{\prime N b} \quad \alpha=1,2
$$

In general, the shear stress components across the interface do not have to be equal to the macroscopic ones as required in the relaxed model. As a result, the model accounts for local equilibrium and compatibility at the interface between two crystals. However, one expects that the average response of composite grains to be equal to the aggregate response. To do so, the global boundary conditions are imposed by relating each composite grain response to the macroscopic behavior of the aggregate as used in the viscoplastic self-consistent scheme ${ }^{8}$.

The basic use for the viscoplastic self-consistent scheme is to solve for the heterogeneous plasticity response for given boundary conditions. Most metals possess anisotropic single crystal properties. Such anisotropy is coupled with the heterogeneity in response, which usually arises from the difference in orientation of grains within a polycrystalline aggregate. In the present setting, each composite grain is treated as a viscoplastic ellipsoid embedded in a Homogeneous Equivalent Medium (HEM). Both individual grains and HEM have uniform properties, where the latter represents the aggregate by averaging the response of all composite grains. 
One is able to allow for local deviation of crystal strain rate and stress from the overall strain rate and stress using the widely known Eshelby solution for a heterogeneous medium. The interaction equation is expressed as:

$$
D-\bar{D}=\tilde{M}:\left[\sigma^{\prime}-\bar{\sigma}^{\prime}\right]
$$

where overbars indicate aggregate averages and $\tilde{M}$ is the interaction matrix written as:

$$
\widetilde{M}=(I-S)^{-1}: S: \bar{M}
$$

$S$ is the Eshelby tensor and $\bar{M}$ is the overall tangent plastic modulus. It is clear that the interaction modulus controls the flexibility allowable for each crystal to plastically deform with respect to the surroundings. For the composite grain model, the response of both crystals is coupled as expressed in Eqs. 1 and 2. An additional step is required to compute the plastic compliance for the composite grain. It has been shown by Lebensohn that the plastic compliance can be computed from ${ }^{9}$

$$
M^{c g}=\left(f^{C u} M^{C u}: A+f^{N b} M^{N b}\right):\left(f^{C u}: A+f^{N b}: I\right)^{-1}
$$

where the matrix $A$ is a function of the individual plastic compliance and the boundary conditions applied at the interface.

By applying the composite grain model to the self-consistent method, it is possible now to consider the local interaction at the interface between two grains ( $\mathrm{Cu}$ and $\mathrm{Nb}$ ) and the global interaction between each composite grain and the aggregate. The current model is used to simulate the macroscopic deformation behavior of the nanoscale $\mathrm{Cu} / \mathrm{Nb}$ multilayers as discussed in section $1 \mathrm{~B}$.

\section{B. Texture evolution in $\mathrm{Cu} / \mathrm{Nb}$ multilayers}

A recent study by Anderson et al. investigated the rolling texture of $\mathrm{Cu} / \mathrm{Nb}$ polycrystalline multilayers ${ }^{10}$. Texture data was supplied by Dr. A. Misra of LANL. The initial individual layer thickness ranges from $75 \mathrm{~nm}$ to $1 \mu \mathrm{m}$. The $\mathrm{Cu} / \mathrm{Nb}$ multilayers were rolled to $50 \%$ thickness reduction. It was reported that the layers with relative large initial thickness exhibit a typical bulk rolling texture for both $\mathrm{Cu}$ and $\mathrm{Nb}$. On the other hand, layers with relative small initial thickness were observed to exhibit a unique rolling texture. The Kurdjumov-Sachs orientation relationship across the $\mathrm{fcc} / \mathrm{bcc}$ interfaces, $<110>\mathrm{Cu} / /<111>\mathrm{Nb}$, was indicated for the as-deposited structure. The measured rolling textures indicated that the Kurdjumov-Sachs relation was lost for the 'thick' layers, while it was preserved to a large degree for the 'thin' layers.

The above experimental texture evidence strongly suggests that there is a change in deformation and strengthening mechanism as the layer thickness is reduced to several nanometers. At this scale, it is believed that the $\mathrm{Cu} / \mathrm{Nb}$ interface plays a key role in affecting the texture development during rolling. In this work, a polycrystal plasticity model is developed to study the unique rolling texture of nanoscale $\mathrm{Cu} / \mathrm{Nb}$ multilayers. The model accounts for the $\mathrm{Cu} / \mathrm{Nb}$ interface by considering a composite grain as the representative volume element of the aggregate. Each grain is divided into $\mathrm{Cu}$ and $\mathrm{Nb}$ crystals in which the interface is parallel to the rolling plane. In addition, a latent hardening formulation is introduced in the composite grain model to account for the resistance to slip transfer across the interface between $\mathrm{Cu}$ and $\mathrm{Nb}$ 


\section{a}

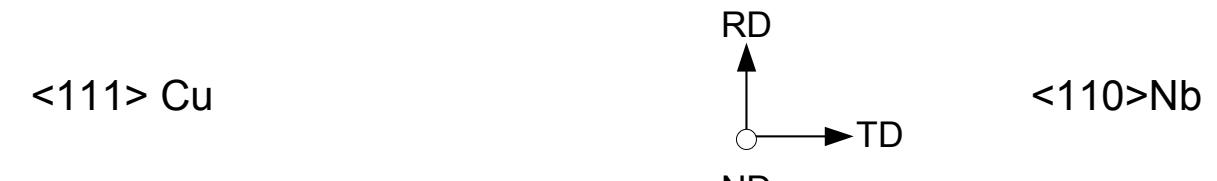

b
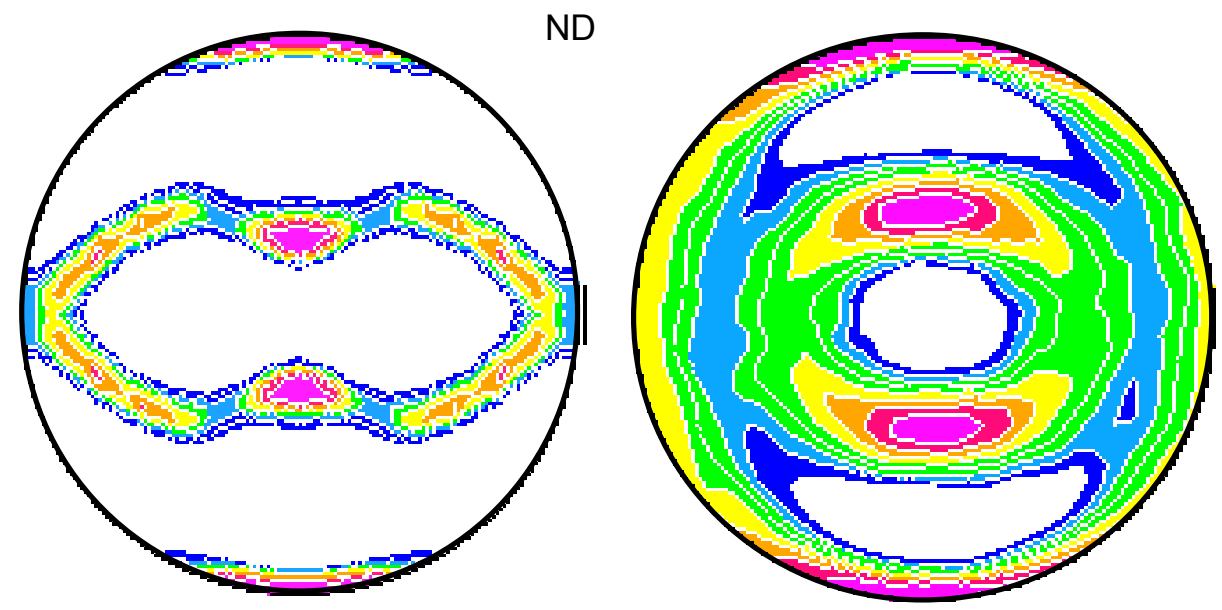

$<111>\mathrm{Cu}$
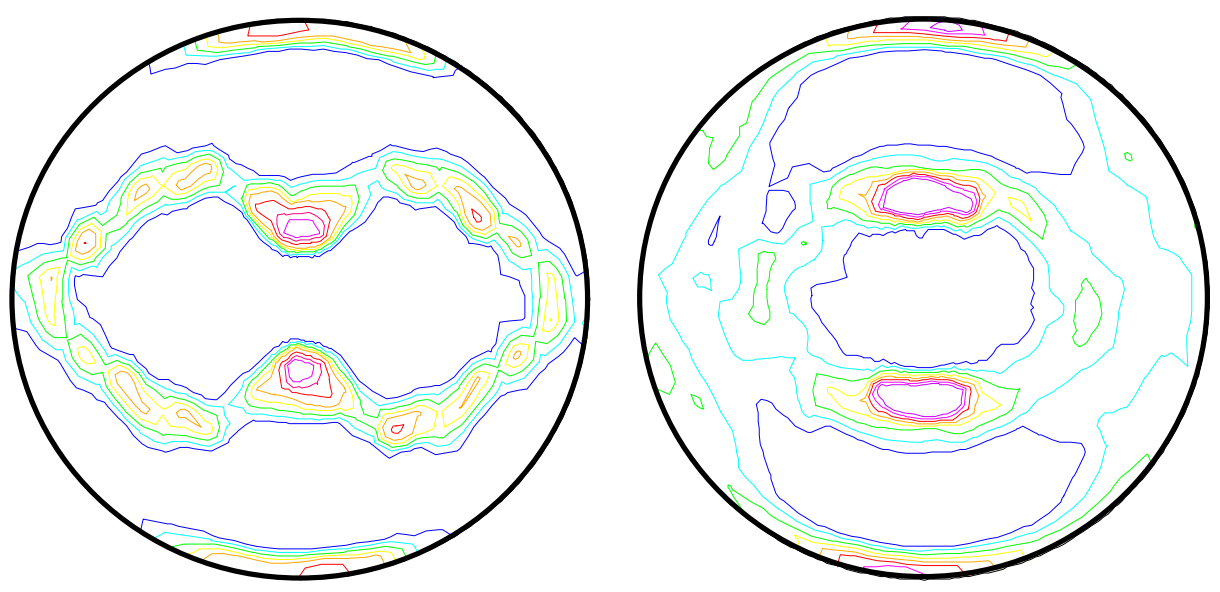

Fig. 2 Rolling Textures of $\mathrm{Cu} / \mathrm{Nb}$ multilayers: Layers were reduced to $50 \%$ of the initial thickness $\left(\varepsilon_{\text {eff }}=0.80\right)$. Initial thickness of individual layers are $1 \mu \mathrm{m}$. a) X-ray diffraction pole figures ${ }^{10}$. b) Simulation pole figures using aggregate of single crystals.

layers. The latent hardening is computed based on storing/annihilating interface dislocations during slip transmission.

To account for the interaction between each composite grain (inclusion) and the medium (polycrystalline sample), the model has been implemented in a viscoplastic self-consistent scheme $^{8}$. In doing so, the model is capable of satisfying compatibility and equilibrium constraints both locally (at interface) and globally (displacement/traction boundary conditions). Due to the presence of thin (flattened) layers, equilibrium at the interface is considered by relaxing the shear components of strain rate at the interface for each crystal in the composite grain.

The VPSC model was first applied to obtain the rolling texture for relatively thick Cu/Nb layers ( $1 \mu \mathrm{m}$ initial layer thickness). The model assumes there is no interaction between individual 
grains. The only interaction allowed is between each grain and the homogeneous equivalent matrix. As a result of having relatively thick layers, one can expect the rotation of each crystal is not affected by its neighbor. Consequently, typical FCC and BCC rolling textures are developed for the $\mathrm{Cu}$ and $\mathrm{Nb}$ phases, respectively. The simulated texture looks very similar to the measured rolling texture of the relatively thick $\mathrm{Cu} / \mathrm{Nb}$ layers as shown in figure 2 . It should be noted that the only restrictions applied to crystal rotation are due to plastic shearing applied on a certain number of slip systems to accommodate crystal deformation and local deviation of crystal deformation response from the macroscopic response expressed.

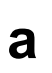

$$
<111>\mathrm{Cu} \quad<110>\mathrm{Nb}
$$

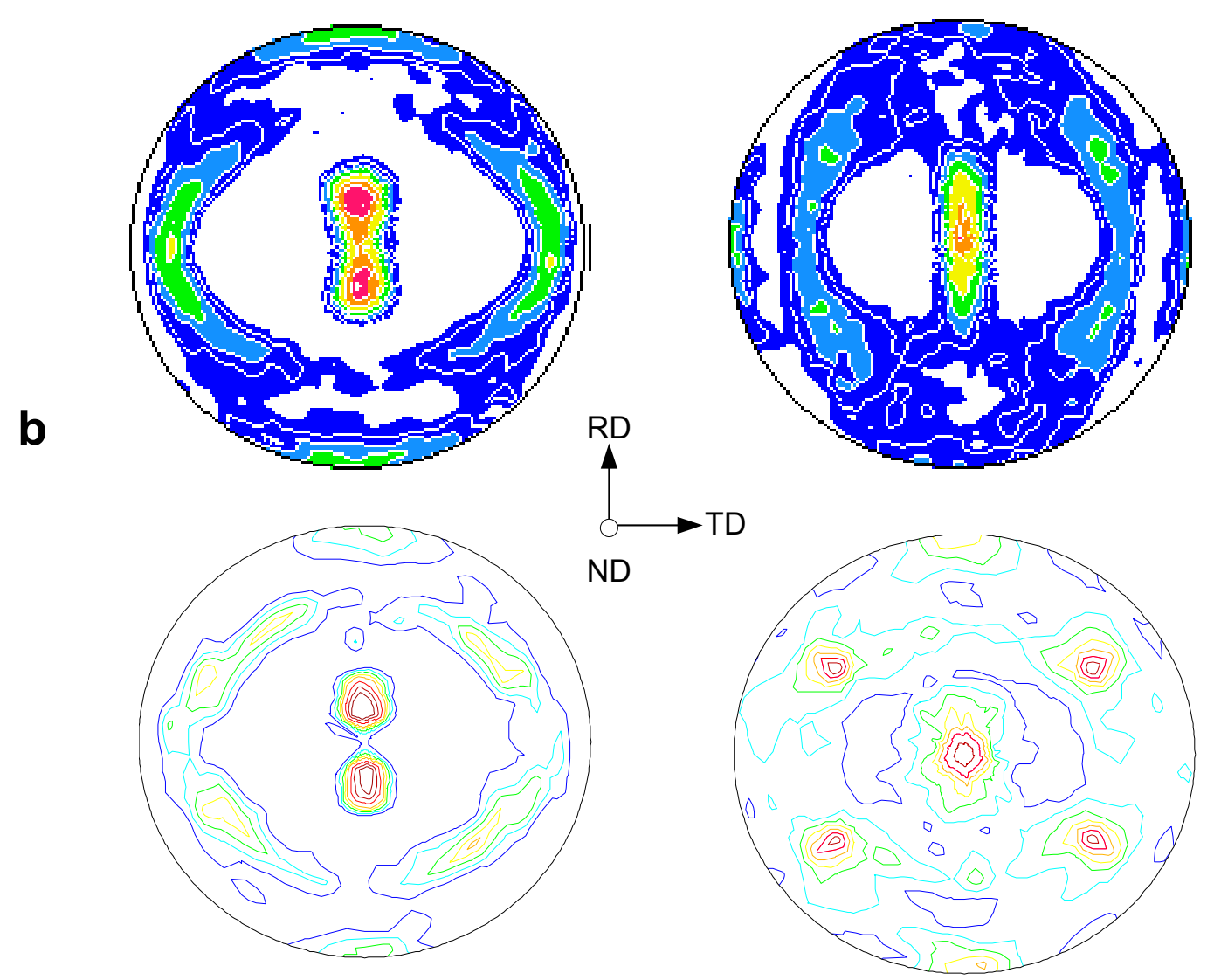

Fig. 3. Rolling Textures of $\mathrm{Cu} / \mathrm{Nb}$ multilayers: Layers were reduced to $50 \%$ of the initial thickness $\left(\varepsilon_{\text {eff }}=0.80\right)$; Initial thickness of individual layers are $75 \mathrm{~nm}$. a) X-ray diffraction pole figures ${ }^{10}$.

b) Pole figures using composite grain model with assumption of latent hardening included.

For the simulation of the nano-layered material, each composite grain is divided into $\mathrm{Cu}$ and $\mathrm{Nb}$ crystals with equal volume fraction. Interfaces between crystals are assumed to be parallel to the rolling plane to satisfy equilibrium and compatibility constraints (Eqs. 3, 4, and 6). Slip system hardening includes a contribution based on net Burgers vector collected in the boundary. The evolution of dislocation content in the boundary is derived from relative rotation between the $\mathrm{Cu}$ and $\mathrm{Nb}$ grains of the bicrystal. The hardening rate is computed based on the this content, assuming a linear arrangement of dislocations in the boundary. [Note that this linear arrangement is relevant to boundaries demonstrating high mobility, as is the case for the $\mathrm{Cu} / \mathrm{Nb}$ system.] Hardening is developed relative to the specific slip system contribution to 
boundary content: slip systems parallel to the boundary remain "soft", while other slip systems harden according to the rate at which they introduce dislocations into the boundary. Figure 3 shows the rolling textures for the nanoscale layers using the modified composite grain model with the latent hardening effect included. Accurate prediction is given considering only these effects of boundary equilibrium and the "latent" hardening due to "excess" boundary content is included. This represents a major advance in our predictive capabilities.

\section{C. Effect of twin boundaries on stress response in polycrystal Ag}

The specific problem being considered is summarized in the stress-strain response shown in figure 4 for fine- ( $2 \mu \mathrm{m}$ grain size) and coarse-grained $(20 \mu \mathrm{m}$ grain size) silver containing annealing twins. Several differences are apparent:

1. the fine-grained material has a higher yield strength than the coarse grained material;

2. the coarse-grained material appears to have a distinct yield point although closer inspection of the stress-strain curve shows a departure from linear behavior at low levels of strain, fig. $4 b$;

3. the presence of a region of uniform elongation with a low work hardening rate exists in the fine-grained material but not in the coarse-grained material;

4. $\quad$ three distinct work hardening stages exist, fig 4c.
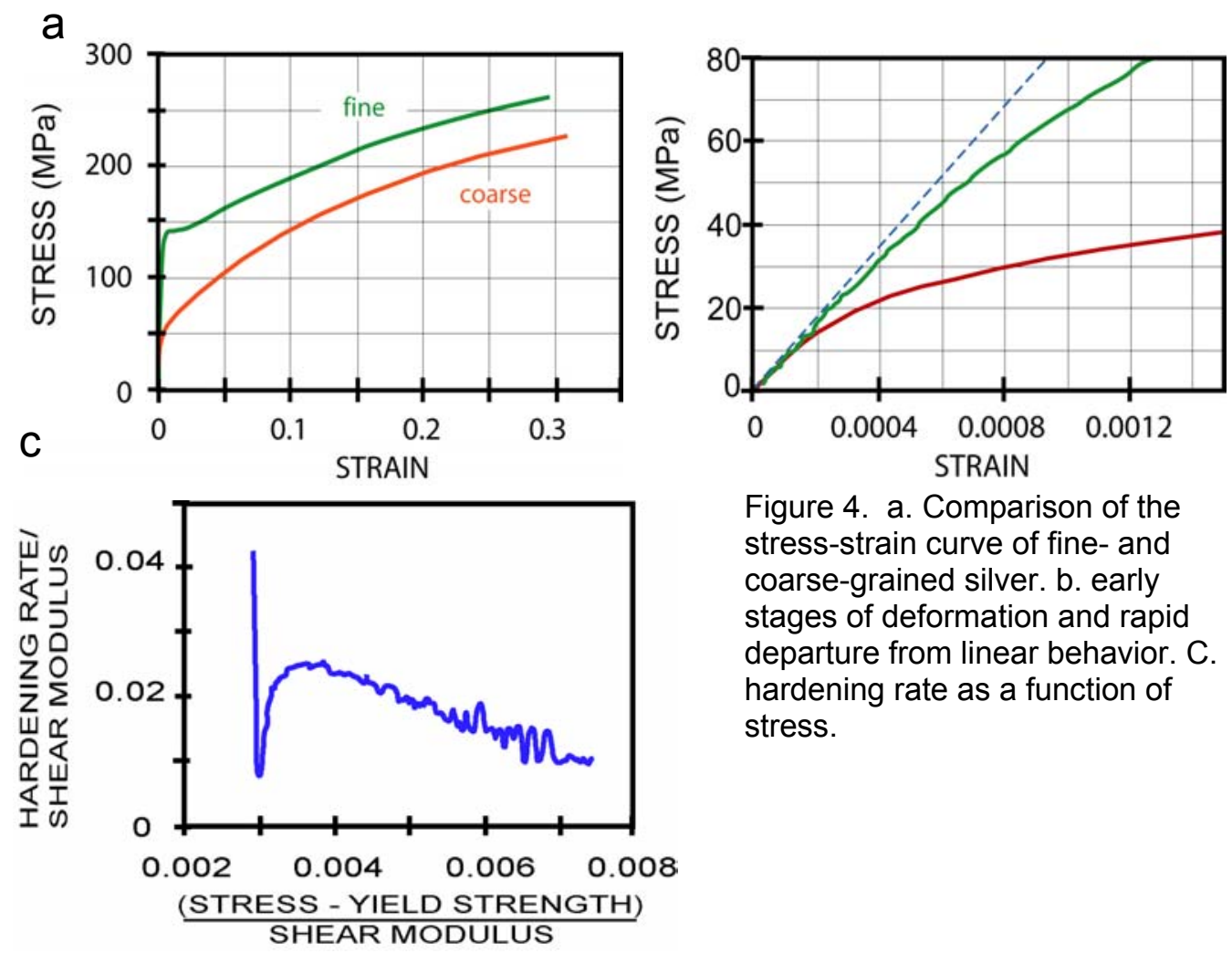

Figure 4. a. Comparison of the stress-strain curve of fine- and coarse-grained silver. b. early stages of deformation and rapid departure from linear behavior. C. hardening rate as a function of stress. 

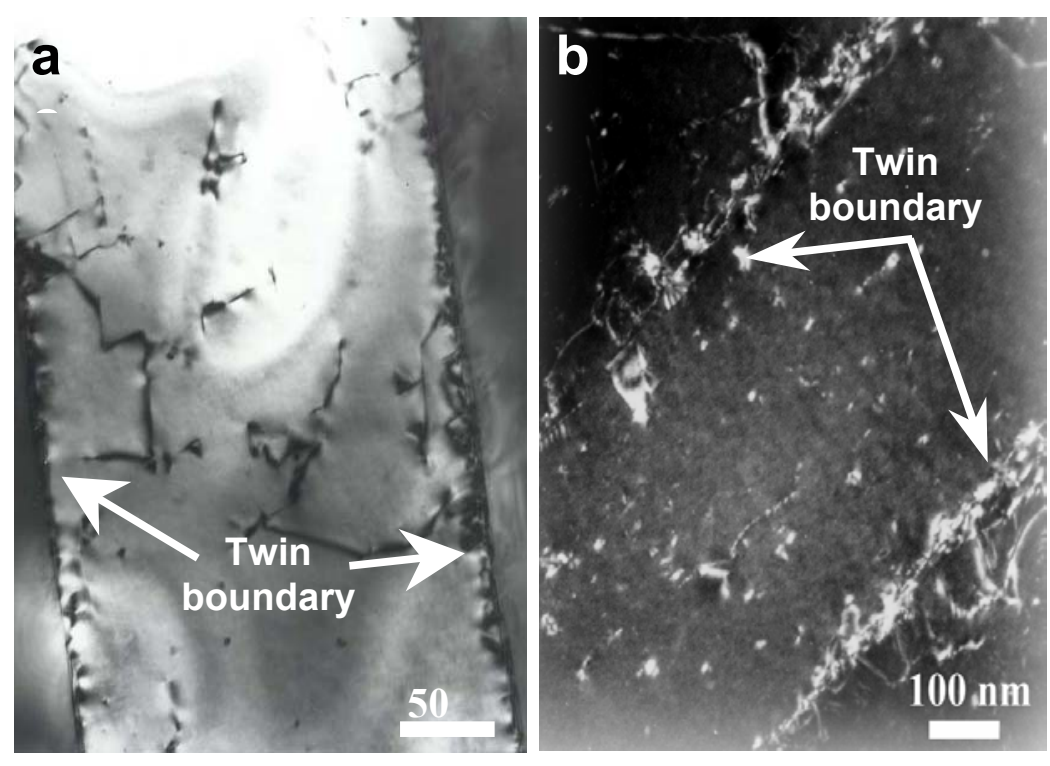

Figure 5. Initial microstructure of the fine-grained material (a) bright-field image and (b) weak-beam dark field image.

The grain size variants were prepared by Dave Korzekwa (LANL) through a process combining rolling and subsequent annealing. In the fine-grained material this process resulted in a microstructure consisting of annealing twins within a matrix that was relatively free of dislocations, figs. $5 \mathrm{a}$ and $5 \mathrm{~b}$. The annealing twin boundaries contain dislocations both within the boundary and attached to it. The question then arises as to the role this microstructure has in determining the observed macroscopic response and how this can be incorporated in a plasticity model.

The difference in the yield strength between the two materials is attributable to the grain size effect. The other differences are not expected and would not be predicted by current plasticity codes. To determine the microstructural changes associated with the macroscopic response some samples were deformed to just beyond the yield point. In the fine-grained material, the microstructure consisted of regions of high dislocation density, Fig 6a, and, unexpectedly, regions with "clean" twin boundaries and with thin deformation twins, Fig. $6 \mathrm{~b}$ and $6 \mathrm{c}$. The clean twin boundaries may reflect strain-induced recovery of the initial microstructure but the presence of locally high dislocation densities and deformation twins suggest the annealing twins are the sources for slip dislocations and for twinning dislocations.

The role of the annealing twins in the generation of slip and twinning dislocations was verified by redeforming the deformed material in situ in the transmission electron microscope and directly observing the deformation modes. These experiments revealed the importance of the preexisting annealing twins as sources for slip and for twinning dislocations, and as barriers to the propagation of slip. Figure 7a shows the emission of partial dislocations from a twin boundary in response to dislocation activity within the twin, and Figure $7 \mathrm{~b}$ shows the creation of deformation twins from annealing twin boundaries. The series of images in Figure 8 show the blockage of slip dislocations by twin boundaries. A dislocation pile-up is formed and with increasing applied load the dislocations eventually break past the barrier. The effectiveness of this barrier and its 

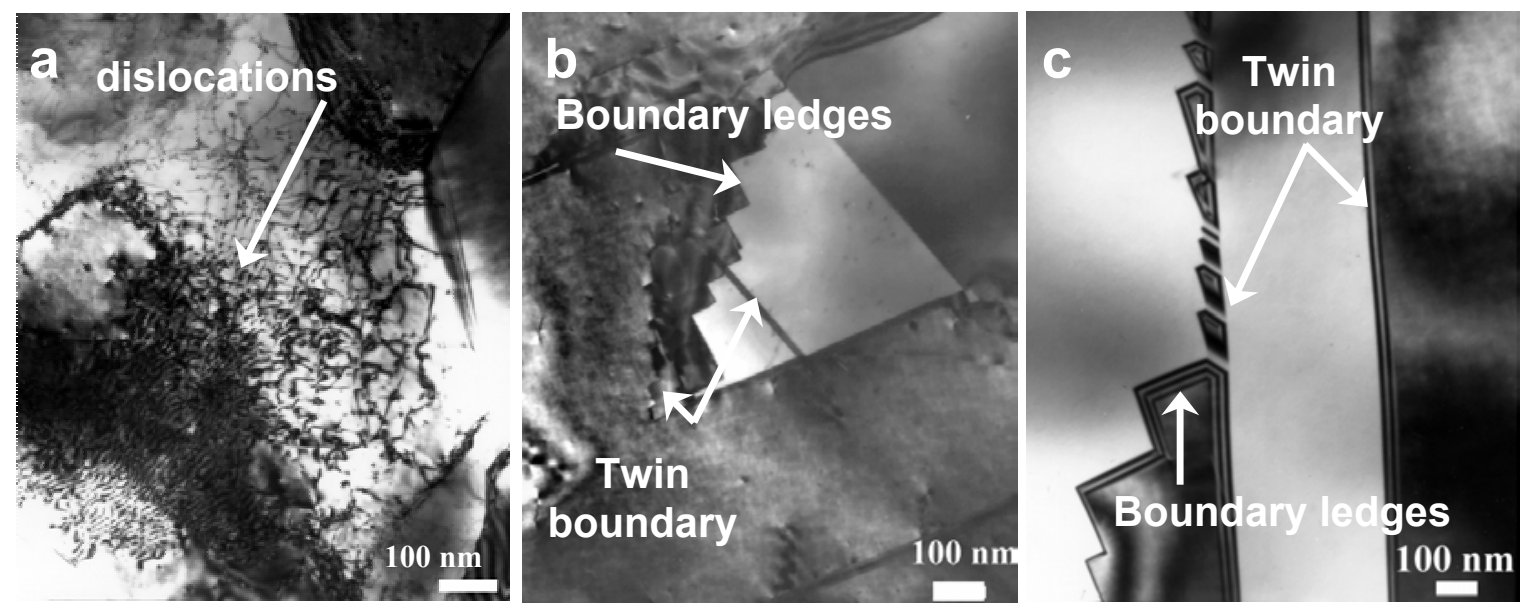

Figure 6. Microstructures observed in fine-grained silver after straining to just beyond the yield point.

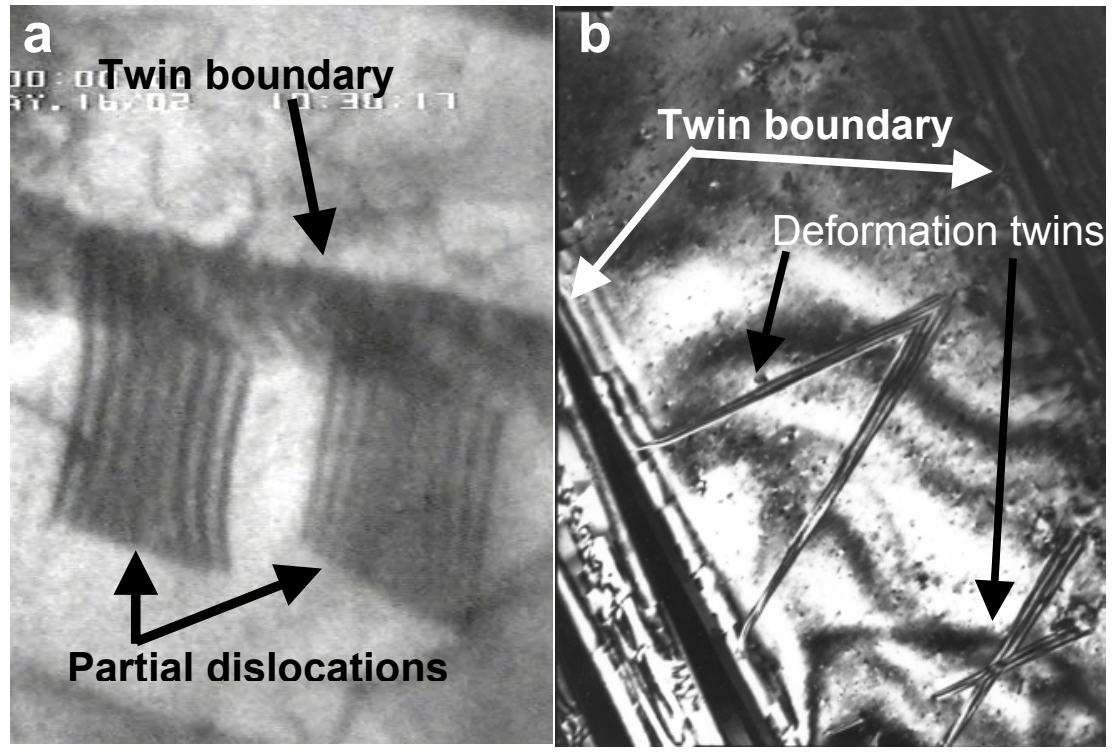

Figure 7. a) emission of partial dislocations and the corresponding stackingfault ribbon from an annealing twin boundary. b) Deformation twins being generated from annealing twin boundaries.

contribution to the strength of the material still needs to be assessed. If it makes a significant contribution, this mechanism will have to be incorporated in plasticity models.

The observations that annealing twin boundaries are sources for slip and twinning dislocations has been incorporated in a polycrystal plasticity model in the form of an inclusion consisting of two crystals separated by a twin boundary. Further, it is assumed that annealing twins are the sources for slip and twinning dislocations.

In the model we complement the usual evolution equation for forest density $\rho_{f}$ with evolution of the mobile dislocation density, $\rho_{m}$. Following Kubin \& Estrin ${ }^{11,12}$ 

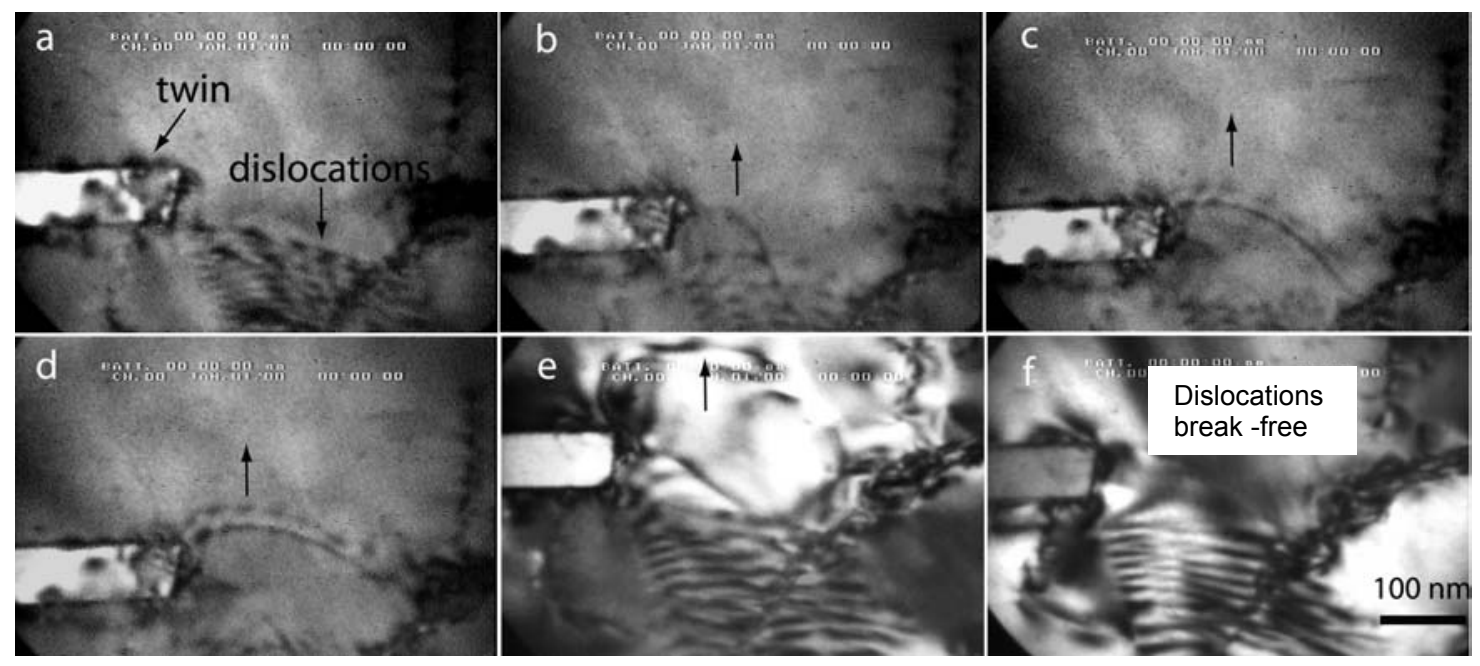

Figure 8. TEM micrographs showing interactions between dislocations and a twin. (a) $t=0$ $\mathrm{s} ;$ (b) $\mathrm{t}=5 \mathrm{~s}$; (c ) $\mathrm{t}=8 \mathrm{~s}$; (d) $\mathrm{t}=9 \mathrm{~s}$; (e) $\mathrm{t}=33 \mathrm{~s}$; (f) $\mathrm{t}=38 \mathrm{~s}$.

$$
\begin{aligned}
& \Delta \rho_{m}=\left(C_{1}-C_{2} \rho_{m}-C_{3} \sqrt{\rho_{f}}\right) \dot{\gamma} \Delta t \\
& \Delta \rho_{f}=\left(C_{2} \rho_{m}+C_{3} \sqrt{\rho_{f}}-C_{4} \rho_{f}\right) \dot{\gamma} \Delta t \\
& \dot{\gamma}=\sum_{s=1}^{n}\left|\dot{\gamma}^{s}\right|
\end{aligned}
$$

where $\dot{\gamma}$ is the collective shear rate over slip systems $s$. Work hardening, through evolution of a strength $\hat{\tau}_{\varepsilon}$, follows from the forest density

$$
\hat{\tau}_{\varepsilon}=\alpha \mu b \sqrt{\rho_{f}}
$$

The strength of the slip system, $\hat{\tau}$, includes both forest hardening and an athermal component, $\hat{\tau}$ as

$$
\hat{\tau}=\hat{\tau}_{0}+\hat{\tau}_{\varepsilon}
$$

Slip system shear rate follows from the usual power law relationship, but includes the effect of the evolving mobile density,

where $P^{s}$ is the Schmid tensor.

$$
\begin{aligned}
& \dot{\gamma}^{s}=\rho_{m} b v_{0}\left(\frac{\tau^{s}}{\hat{\tau}}\right)^{m} \\
& \tau^{s}=P^{s} \cdot \sigma^{c}
\end{aligned}
$$

A comparison of the predicted and experimental response is shown in figure 9. Clearly, the main features of the stress-strain curve have been captured especially just beyond the yield point. The applicability of the approach is being tested against over systems such as the response of copper, which shows a similar stress-strain relationship but without deformation twinning. 


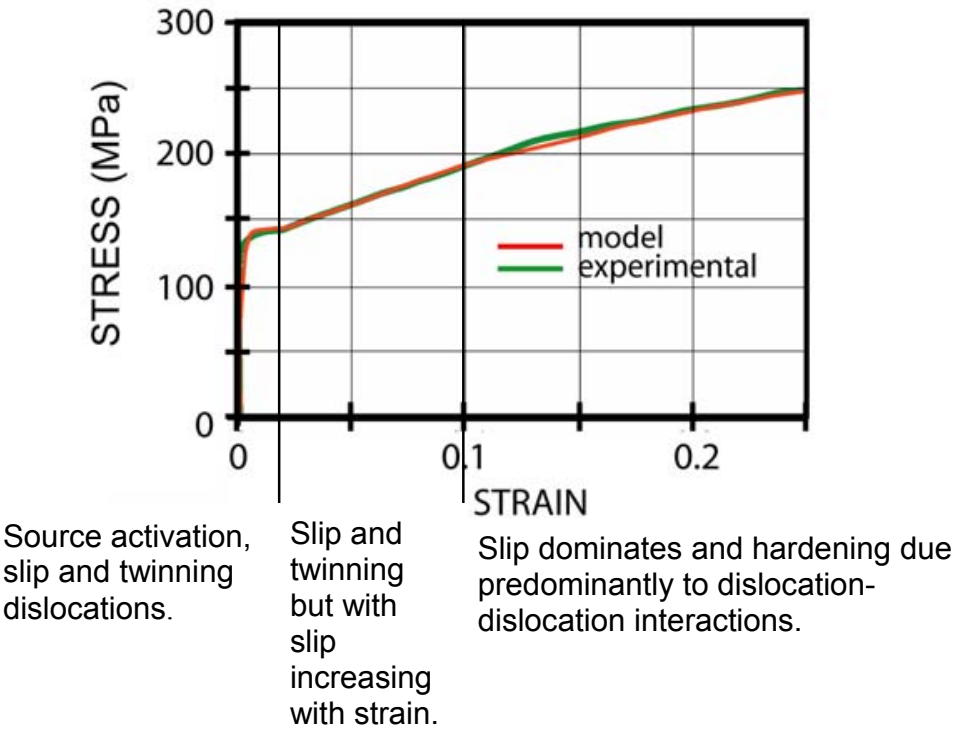

Figure 9. Comparison of the experimental and simulated stress-strain relationships.

\section{Development of dynamic testing capability.}

The high strain rate part of this program consists of mechanical characterization of the materials of interest $\left(\mathrm{Cu}, \mathrm{Zr}, \mathrm{V}\right.$ etc.) under a wide range of strain rates $\left(10^{-3} / \mathrm{s}-10^{7} / \mathrm{s}\right)$. Quasi-static rates are achieved using traditional servo-hydraulic loading devices. Dynamic loading experiments with strain rates in the range $10^{2} / \mathrm{s}-10^{4} / \mathrm{s}$ are conducted using a Split Hopkinson Pressure Bar (SHPB) set-up. A laser loading facility is under development for experiments involving loading rates in the range $10^{4} / \mathrm{s}-10^{7} / \mathrm{s}$. These are new capabilities we are developing as part of this effort.

The setup of the Split-Hopkinson Pressure Bar test facility has been completed and initial experiments have been performed to test the capabilities. Dynamic experiments have been conducted thus far on OFHC Cu and 2024-T6 Al (as model FCC materials), and Zr (an HCP material). These experiments have focused on obtaining the stress strain response of the materials up to saturation stress levels. Figure 10a,b,c shows a set of stress strain curves for $\mathrm{Cu}, \mathrm{Al}$ and $\mathrm{Zr}$ respectively. The first two figures include a quasi-static stress strain curve along with three dynamic response curves obtained in the SHPB. The arrows in the figure indicate the strain level (and time $t_{h}$ ) at which the loaded specimen achieves homogenization conditions. This time is obtained experimentally by comparing the measured forces at the specimen/incident bar and specimen/transmitted bar interfaces. Some of the stress-strain curves shown in Fig. 10b and 10c have been obtained using a pulse shaper. In this configuration of the SHPB, a thin Cu disk is placed at the impact end of the incident bar. The disk, which yields upon impact, produces an incident pulse with a more gradual rise time to peak loading. The purpose of these tests was to validate the experimental setup, the implementation of pulse shaping techniques, and to obtain some preliminary dynamic stress strain curves for materials of interest ( $\mathrm{Cu}$ and $\mathrm{Zr}$ ). 

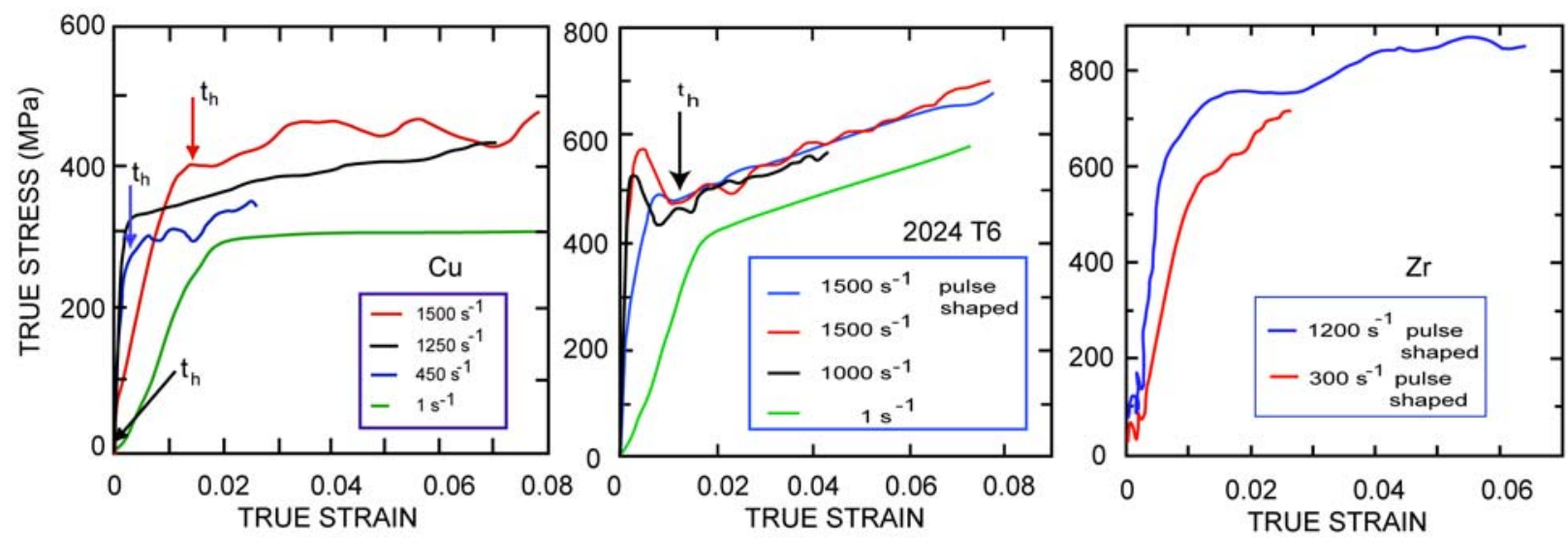

Figure 10. Uniaxial compression true stress strain curves for (a) $\mathrm{Cu}$, (b) Al and (c) $\mathrm{Zr}$.

Figure 11 shows a comparison between the dynamic stress strain response of OFHC Cu obtained in the present study and that found in several sources in the literature ${ }^{13-16}$. As can be seen the present results agree very well with those of Bragov and Lomunov ${ }^{16}$ while both are quite different from those in the other works. The reason for this difference is not clear at present. Although all studies have used OFHC $\mathrm{Cu}$ the microstructural details, specifically grain size, are not known in each case. It is believed that the effect observed could be the result of the fine grain structure of the $\mathrm{Cu}$ used in these tests.

\section{Laser loading experiments}

In terms of this project, a novel laser loading device is being developed that would be capable of applying (estimated) strain rates between $10^{4} / \mathrm{s}$ and $10^{7} / \mathrm{s}$ to the materials of interest. The methodology is based on the laser loading spallation experiments outlined in Gupta et al. ${ }^{17,18}$ The set-up is shown schematically in Figure 12: a high power short duration laser pulse is incident on a thin energy absorbing layer deposited on the surface of a substrate (glass, silicon or quartz). The layer rapidly expands and since it is confined on one side by a confining layer (here waterglass which is transparent to the laser light used) a

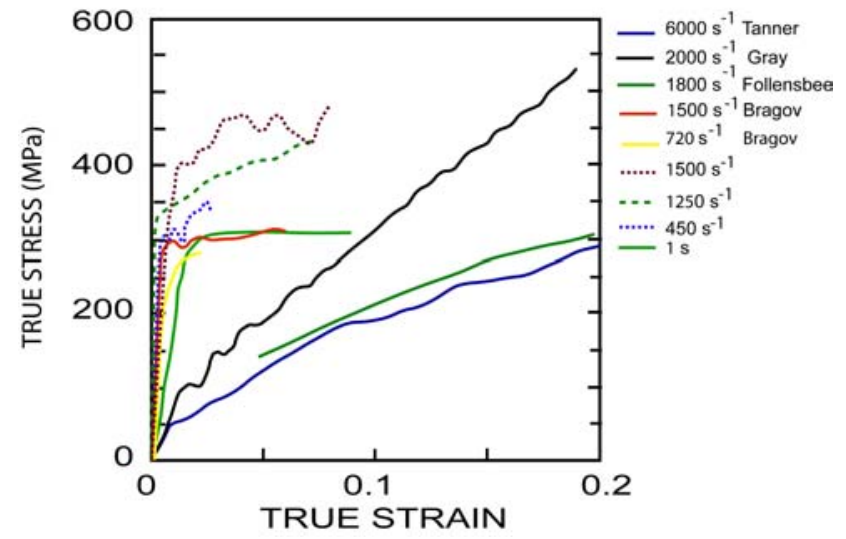

Figure 11: Comparison of present results for OFHC Cu dynamic response with those in literature ${ }^{13-16}$.

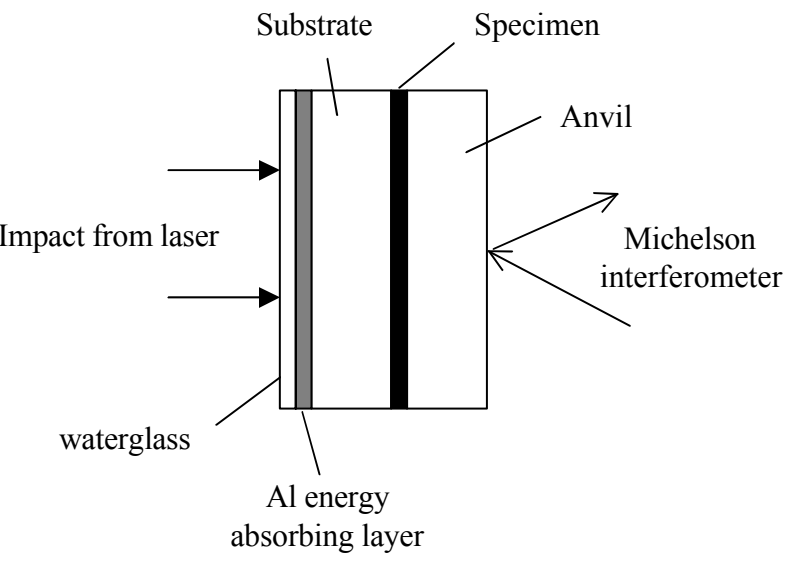

Figure 12: Schematic of laser loading experiment. 
stress wave is launched into the substrate. In the work of Gupta et al. ${ }^{17,18}$ this wave, which can become a shock depending on the substrate material and the intensity of loading, was used to study interface spallation strength. In the present work an anvil is placed behind the sample so that it is sandwiched between two elastic substrates forcing it to yield. A Michelson interferometer is used to measure the free surface velocity on the back surface of the transmitted anvil. Simple one dimensional wave propagation calculations can then yield the stress at the interface between the anvil and sample.

A Spectra Physics quanta Ray Lab 190-10 Nd:YAG laser with a peak energy per pulse of $1 \mathrm{~J}$ and pulse width (FWHM) of 11.7 ns has been obtained for this work. Thus far we have performed a calibration study of laser induced stress wave propagation in glass substrates (and are now in the process of testing silicon and quartz substrates). Soda-lime glass slides were coated with aluminum on both the front and back surfaces using an electron beam evaporator. A $500 \mathrm{~nm}$ layer of aluminum was deposited on one side to act as the energy absorbing layer. The other side was coated with a $100 \mathrm{~nm}$ thick layer to act as a reflecting surface for the Michelson interferometer. The absorbing layer was then coated with a 30 micron thick layer of waterglass which acts as a confining layer, and increases the amplitude of the stress pulse in the substrate. For the results presented below, the beam was focused to a $5 \mathrm{~mm}$ spot on the absorbing layer. However, higher fluence (= energy/area) could be achieved by focusing the beam down to $2 \mathrm{~mm}$. The Michelson interferometer, which is sensitive to out-of-plane displacements, compares the optical path length between a reference beam and a beam reflected off the specimen surface. The beams are combined onto a photodetector: (ElectrOptic technologies ET-4000 with $10 \mathrm{GHz}$ bandwidth and 35 ps rise time) which records a maximum intensity when the beams interfere constructively and minimum when they interfere destructively. The signal is recorded on a Tektronix $694 \mathrm{C}$ digital oscilloscope (3 $\mathrm{GHz}$ bandwidth, $10 \mathrm{GS} / \mathrm{s}$ ). A single mode laser is needed as the illumination source for the interferometer. In the present work we use a Crystalaser DPSS single longitudinal mode laser operating at $532 \mathrm{~nm}$ with $25 \mathrm{~mW}$ power.

Figure 13a shows a typical signal from the interferometer recorded during loading of a single glass substrate. The peaks and valleys, corresponding to constructive and destructive interference respectively, are clearly visible. From this signal one can obtain a record of the free surface (i.e., the one opposite the impact end) velocity, as shown Figure 13b. Finally, differentiating the displacement (either by curve fitting or numerically if the sampling rate is sufficient) provides the free surface velocity, and consequently stress, shown in Figure 13c. Figure 14 shows the results of a calibration study in which the fluence of the loading pulse was varied by changing the laser power and keeping the impact area constant at $5 \mathrm{~mm}$. As can be seen it is possible to control the pulse magnitude quite well in this fashion.

In the immediate future it is planned to perform similar incident calibration tests on silicon and quartz substrates. Subsequently $\mathrm{Cu}, \mathrm{Zr}$ and $\mathrm{V}$ will be tested in the configuration shown in Figure 12. A gripping fixture to hold the $5 \mathrm{~mm}$ diameter specimens has been designed and constructed. As seen above, the loading and free surface diagnostics have also been implemented. However, in order to obtain accurate measurements of both stress and strain in the sample we also need a measurement of the pulse reflected from the specimen back into the substrate. One way of obtaining this measurement, at least for the case of quartz substrates, is by making use of the piezoelectric properties of the quartz itself. Graham et al. ${ }^{19}$ showed that using measurement of the incident and reflected pulses in the quartz. (In fact before widespread use of displacement or velocity interferometry this was the most common way of measuring the substrate stress.) However, in the work of Graham et al. ${ }^{19}$ and since then (e.g. Gupta and Epstein ${ }^{18}$ ) this method has been used for measuring the incident stress pulse in the calibration 

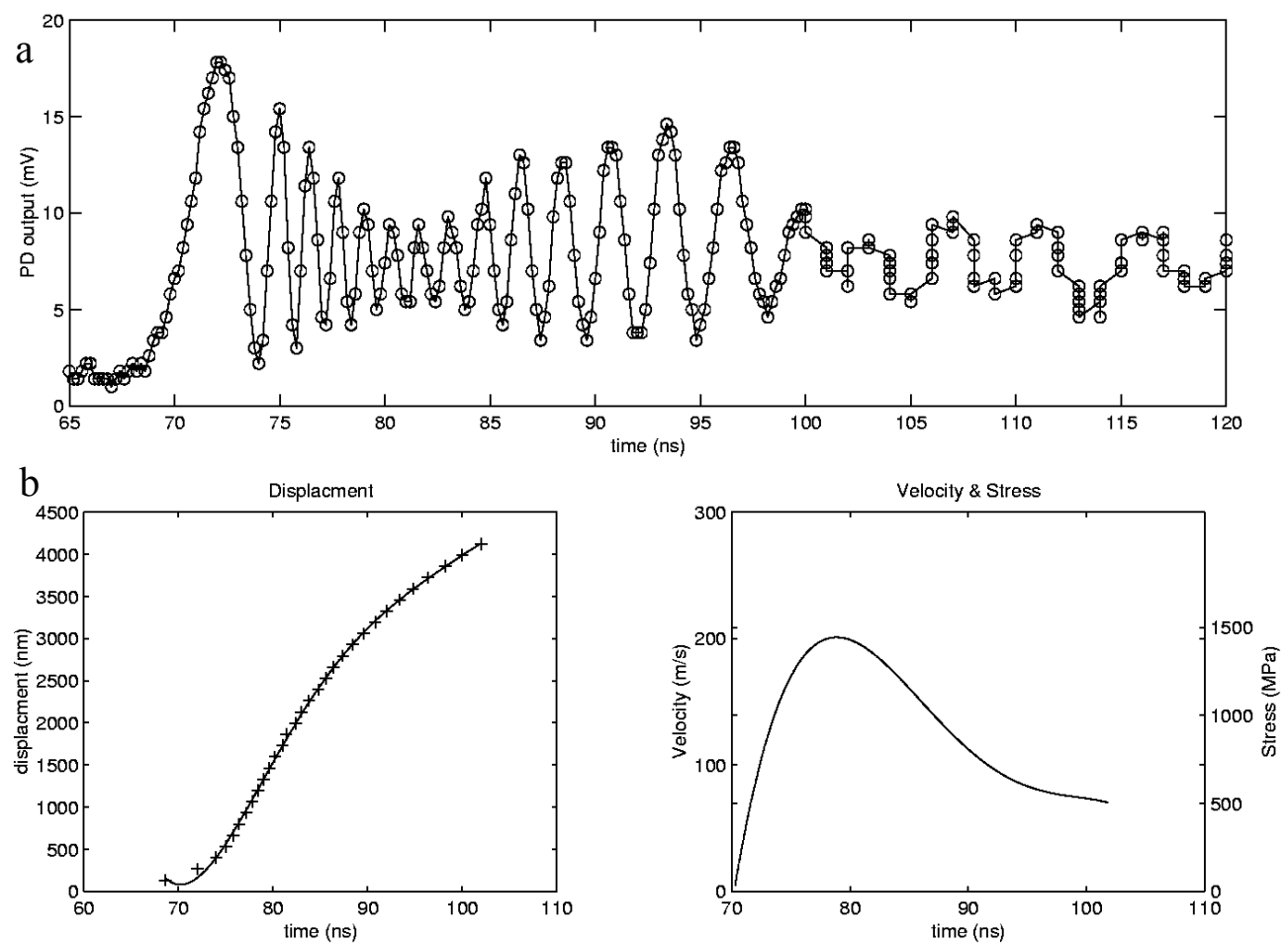

Figure 13: Typical result from laser loading experiment on glass substrate.

of a free standing quartz disk (much like the results of Figure 13 that were obtained using interferometry). In the present work, however, it is the incident and reflected pulses when we place a metal sample after the quartz substrate that need to be recorded. Therefore, the quartz surface is no longer traction free and the reflected pulse will be altered depending on the impedance mismatch, $\alpha=\frac{\rho_{2} c_{2}}{\rho_{1} c_{1}}$ where $\rho$ is density and $\mathrm{c}$ dilatational wave speed, between the sample and the quartz substrate. The analysis of Graham et al. ${ }^{19}$ has therefore been extended to include a possible impedance mismatch between quartz and sample. The main result is shown graphically in Figure 14. The average polarization of the quartz < $P>$ changes only when there is a change of strain with it. Consequently, it can be shown that the resulting current $i(t)$ recorded by the oscilloscope is proportional to the free surface velocity. Both are shown schematically in Figure 14 with the traction free case described by Graham et al. ${ }^{19}$ corresponding to the impedance mismatch of $\alpha=$ 0 , while $\alpha=1$ and $\alpha=\infty$ correspond to the impedance matched and built-in cases, respectively. From the theoretical results of Figure 14, it appears possible, in a set-up involving a quartz substrate, to obtain a measure of the pulse

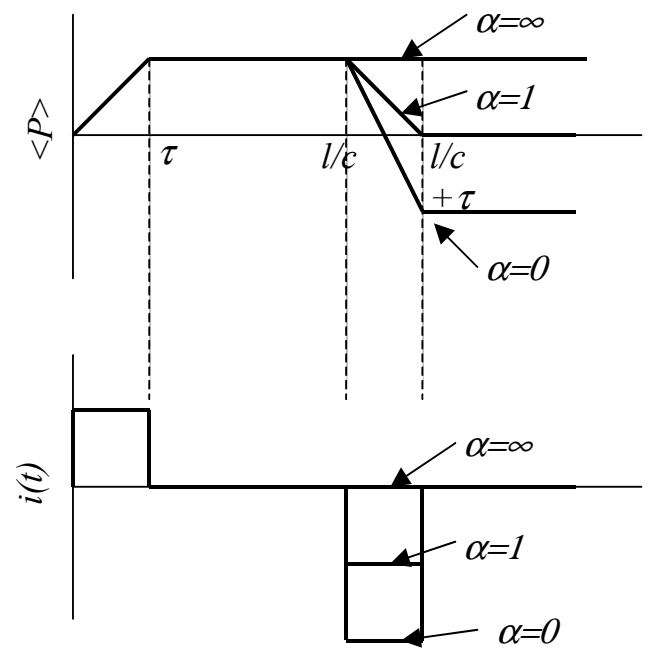

Figure 14. Results of theoretical analysis predicting the piezoelectric response of a quartz substrate in contact with and impedance mismatched material. 
reflected from the sample. This, in addition with the measurement of free surface velocity of the anvil should allow for complete determination of the state of the sample. We have already purchased a number of $5 \mathrm{~mm}$ X-cut piezoelectric disks and will be testing them in the near future.

\section{Manuscripts and Presentations}

This work has been presented at the following meetings

1. "Revealing the microstructural processes controlling deformation and fracture." I. Robertson, Mechanics Congress, New Mexico, July 2003. Invited talk.

2. "Effect of residual dislocations due to slip transmission at twin boundaries:Incorporation in Viscoplastic Self-Consistent Plasticity". K. Al-Fadhalah LANL, Los Alamos, March 2003.

3. "Microplastic Processes Developed in Pure Ag with Mesoscale Annealing Twins" Mechanics and Materials ASME Conference, Scotsdale Arizona, June 2003.

4. "Modeling texture evolution during rolling of $\mathrm{Cu}-\mathrm{Nb}$ multilayered system" K. Al-Fadhalah LANL Meeting of the BES program on Nanoscale Metallic Multilayers Los Alamos,NM., Sept, 2003.

5. "Microplastic Processes Developed in Pure Ag with Mesoscale Annealing Twins" K. AlFadhalah Fall Meeting of TMS: Materials Science and Technology, Chicago II 2003.

6. Poster presentation by the graduate students at the annual review of the ASCl level1 Center at the University of Illinois. K. Al-Fadhalah, C. Smith, B. Miller, Jamie Kimberley Henry Padilla Satya Varadhan

7. Multi-scale modeling of materials, January, 2004. Meeting organized by LLNL. Ian Robertson, John Lambros and Armand Beaudoin.

As yet no publications have resulted from the work, although two are in preparation

1. Microplastic processes in pure silver with mesoscale annealing twins, K. Al-Fadhalah, C. M. Li, A. J. Beaudoin, and I. M. Robertson, University of Illinois and C. N. Tome, Los Alamos National Laboratory. Anticipated submission date January 2004. The basis of this paper is presented in key achievement 1.

2. Multi-layered material paper - Anticipated submission date January 2004. The basis of this paper is presented in key achievement 1.

\section{Interactions with National Laboratories}

\section{Visits to National Laboratories}

Al-Fadhalah and Beaudoin Los Alamos National Laboratory, 24 March 2003 to 6 April 2003; 2 Sept 03 to 4 Sept 03.

J. Lambros, Livermore National Laboratory, Feb 2003.

2. Visits by lab people to UIUC.

Geoff Campbell, Livermore National Laboratory 18 Dec. 2003

Carlos Tomé, Los Alamos National Laboratory. 


\section{References}

1 T. C. Lee, I. M. Robertson, and H. K. Birnbaum, Ultramicroscopy 29, 1-4 (1989).

2 T. C. Lee, I. M. Robertson, and H. K. Birnbaum, Metallurgical Transactions A-Physical Metallurgy \& Materials Science 21A, 2437-47 (1990).

3 W. A. T. Clark, R. H. Wagoner, Z. Y. Shen, T. C. Lee, I. M. Robertson, and H. K. Birnbaum, Scripta Metall. et Mater. 26, 203-206 (1992).

$4 \quad$ J. Shirokoff, I. M. Robertson, and H. K. Birnbaum, in Defect Interface Interactions; Vol. 1994 (Materials Research Society, Pittsburgh, PA, USA, 1994), p. 263-272.

$5 \quad$ M. F. Savage, J. Tatalovich, M. Zupan, K. J. Hemker, and M. J. Mills, Mater.Sci. and Engin. A 319-321, 398-403 (2001).

$6 \quad$ J. M. K. Wiezorek, X. D. Zhang, W. A. T. Clark, and H. L. Fraser, in Proceedings of the 1996 MRS Fall Meeting, Dec 2-5 1996; Vol. 460 (Materials Research Society,

Pittsburgh, PA, USA, Boston, MA, USA, 1997), p. 231-236.

7 R. G. Hoagland, T. E. Mitchell, J. P. Hirth, and H. Kung, Phil. Mag. A 82, 643 - 664 (2002).

$8 \quad$ R. A. Lebensohn and C. N. Tome, Acta Metall. et Mater. 41, 2611-2624 (1993).

$9 \quad$ R. Lebensohn, in Modelling and Simulation in Materials Science and Engineering. Proceedings of the 1998 Gilles Genova Memorial Symposium on Multi-scale Modelling of Mechanical Properties of Materials.; Vol. 7 (Institute of Physics Publishing, Bristol, Engl, Autrans, France, 1999), p. 739-746.

P. M. Anderson, J. F. Bingert, A. Misra, and J. P. Hirth, Acta Mater. 51, 6059 -6075 (2003).

11 L. P. Kubin and Y. Estrin, Acta Metall. 33, 397-407 (1985).

L. P. Kubin and Y. Estrin, Acta Metall. 38, 697-708 (1990).

A. B. Tanner and D. L. McDowell, Inter. J. Plasticity 15, 375 (1999).

G. T. Gray, (2003).

P. S. Follansbee and U. F. Kocks, Acta Metall. 36, 81-93 (1988).

A. M. Bragov and A. K. Lomunov, Inter. J. Impact Engr. 16, 321-330 (1995).

V. Gupta, A. S. Argon, J. A. Cornie, and D. M. Parks, Mater.Sci. and Engin. A 126, 105 117 (1990).

V. Gupta and D. J. Epstein, J. Appl. Phys., 67, 2185-2188 (1990).

R. A. Graham, F. W. Neilson, and W. B. Benedick, J. Appl. Phys., 36, 1775-1783 (1965). 